\title{
Numerical determination of the effective moments of non-spherical particles
}

\author{
Nicolas G Green ${ }^{1}$ and Thomas B Jones ${ }^{2}$ \\ ${ }^{1}$ School of Electronics and Computer Science, Southampton University, Southampton SO17 \\ 1BJ, UK \\ ${ }^{2}$ Department of Electrical and Computer Engineering, University of Rochester, Rochester, \\ NY 14627, USA
}

Received 8 September 2006, in final form 9 September 2006

Published 15 December 2006

Online at stacks.iop.org/JPhysD/40/78

\begin{abstract}
Dielectric characterisation of polarisable particles, and prediction of the forces and torques exerted upon them, relies on the knowledge of the effective, induced dipole moment. In turn, through the mechanism of depolarisation, the induced dipole moment of a particle is strongly dependent upon its shape. Since realistic shapes create modelling difficulties, the 'spherical particle' approximation is often invoked. However, in many cases, including biological dielectric spectroscopy and dielectrophoresis, this assumption is a poor one. For example, human erythrocytes are essentially oblate spheroids with indented sides, while viruses and bacteria often have elongated cigar shapes. Since shape-dependent polarisation both strongly influences the accuracy of conventional dielectric characterisation methods using Maxwell's mixture formula and confounds accurate prediction of dielectrophoretic forces and torques, it is important to develop means to treat non-spherical particles. In this paper, we demonstrate a means to extract the dipole moment directly from numerical solutions of the induced electrostatic potential when a particle is placed in a uniform electric field. The accuracy of the method is demonstrated for a range of particle shapes: spherical, ellipsoidal, truncated cylinders and an approximation of an erythrocyte, the red blood cell.
\end{abstract}

(Some figures in this article are in colour only in the electronic version)

\section{Introduction}

A wide range of particle characterisation, manipulation and separation techniques being developed rely on the interaction of electric fields and the particles. Dielectric or impedance spectroscopy involves the application of low voltage signals to suspensions of particles and the measurement of the response [1-4]. Under a (normally) uniform applied field, the different materials (comprising the particle and the suspending fluid) polarise, resulting in induced effective moments around the particle [5,6]. Spectroscopy in this case involves the detection of the additional polarisation due to the particles. This technique can provide detailed information about the particle size and the internal and surface properties [2-4,7-9] and has been used for the characterisation of a wide range of particle types. Recently, using advanced microfabrication techniques and developments in electronics, single-cell dielectric spectroscopy has been demonstrated for application in high throughput screening $[10,11]$.

Dielectrophoresis is the motion of particles due to the interaction of a non-uniform applied electric field and the moments induced in particles $[5,6,12]$. It can be used for characterisation and also for manipulation and separation [12-17]. Electrorotation [5, 6, 14,17-20] and travelling wave dielectrophoresis $[6,15,16]$ involve, respectively, the rotation and motion induced by electric fields with directional or spatial phase non-uniformity.

Each of these techniques relies on a fundamental understanding of the polarisation mechanism of the particle and the fluid medium in which it is suspended. When an electric field is applied, the different materials polarise, producing charge at all interfaces, particularly the interface between the particle and the fluid. The net charge has opposite sign on either side of the particle, leading first and foremost 
(a)

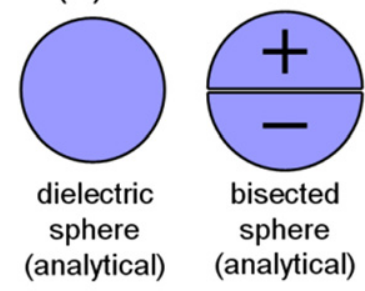

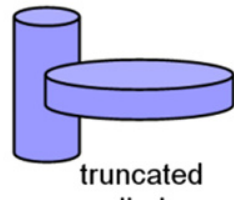

cylinder

(no analytical solution)

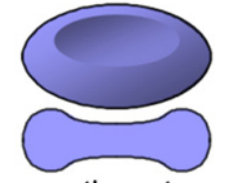

erythrocyte

(no analytical solution)

(b)

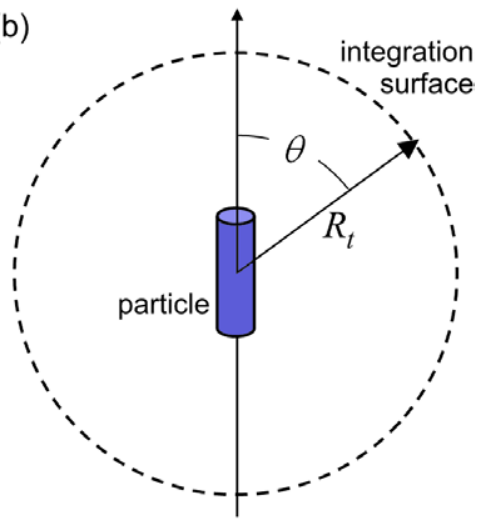

(c)

dipole

$n=1$
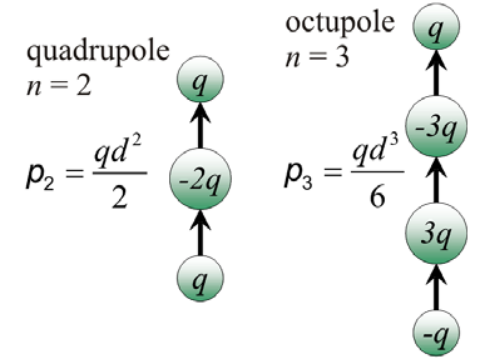

Figure 1. (a) Outline of the different test particles simulated in this paper with an indication of whether or not an analytical representation exists. (b) The arrangement of the spherical surface with radius $R_{\mathrm{t}}$ around the arbitrary particle. (c) Representation of the first three linear multipoles and the simple equation giving the size of the moment

to a net-induced effective dipole moment. For a spherical particle, the solution of the electric field around the particle can be expressed in terms of Legendre polynomials [21-23], demonstrating that the effect of the particle is indistinguishable from a point dipole (and possible higher order moments). This dipole moment depends on the frequency of the applied electric field and the dielectric properties (permittivity and conductivity) of the particle and the fluid [6]. Measurements of impedance in spectroscopy, velocity in dielectrophoresis or rotation rate in electrorotation are all made with the goal of determining the dipole moment of the particle and from there the properties of the particle.

As stated above, for a spherical particle in a uniform field, there is a dipole moment and all higher order moments are zero. The standard assumption in DEP or impedance spectroscopic measurement of particle suspensions assumes that the particle is spherical and all higher order moments are zero. Non-spherical particles can have significant higher order components, and in some cases these moments strongly influence the observable motions. The presence of higher order contributions to the energy stored in the field would lead to errors in estimating the dipole moment of the particle. This problem becomes important when the electric field is highly nonuniform or at high volume fractions, as the field from higher order moments decreases much more rapidly than the dipole.

The force and torque in non-uniform electric fields are affected directly by these higher order moments for nonspherical particles. The first two terms in the dielectrophoretic (DEP) force $\boldsymbol{F}_{\mathrm{DEP}}$ and electrorotational torque $\boldsymbol{\Gamma}_{\mathrm{ROT}}$ are [5]

$$
\boldsymbol{F}_{\mathrm{DEP}}=\mathrm{p}_{1} \cdot \nabla \boldsymbol{E}_{\mathrm{o}}+\frac{1}{2} \mathrm{p}_{2} \cdot \nabla \nabla \boldsymbol{E}_{\mathrm{o}}+\cdots
$$

and

$$
\Gamma_{\mathrm{ROT}}=\mathrm{p}_{1} \times \boldsymbol{E}_{\mathrm{o}}+\mathrm{p}_{2} \times \nabla \nabla \boldsymbol{E}_{\mathrm{o}}+\cdots,
$$

where $\boldsymbol{E}_{\mathrm{o}}$ is the applied electric field, $\mathrm{p}_{1}$ is the dipole moment, $\mathrm{p}_{2}$ is the quadrupole, and so on. Knowledge of higher order moments is therefore essential in calculation, a fact that has led to the development of theories to handle arbitrary higher order forces [24-26] and numerical methods for determining the DEP force accurately for non-spherical particles [27].

This paper presents a straightforward method for calculating the linear electrical moments of arbitrary shaped particles. The method uses the analytical expressions for the higher order potentials along with numerical solutions of these potentials to calculate the values of individual moments. It is applicable for any method of determining the electrical potential and therefore for any shape or type of particle. The accuracy of the method is demonstrated for the range of particle shapes indicated schematically in figure 1(a): spherical, ellipsoidal, truncated cylinders and an approximation of an erythrocyte, the red blood cell (RBC). We find that the dipole moment can be calculated rapidly and accurately for any shape of particle and that moments up to 9th order are easily obtainable.

\section{Background and theory}

Direct extraction of the dipole and higher order moments from numerical solutions for the induced electrostatic potential relies upon a properly weighted integral over a spherical surface centred on the particle with a radius $R_{\mathrm{t}}$ equal to several times the longest dimension of the particle, as shown in figure $1(b)$. The method is completely general, but here we restrict our attention to the case of cylindrically symmetrical 

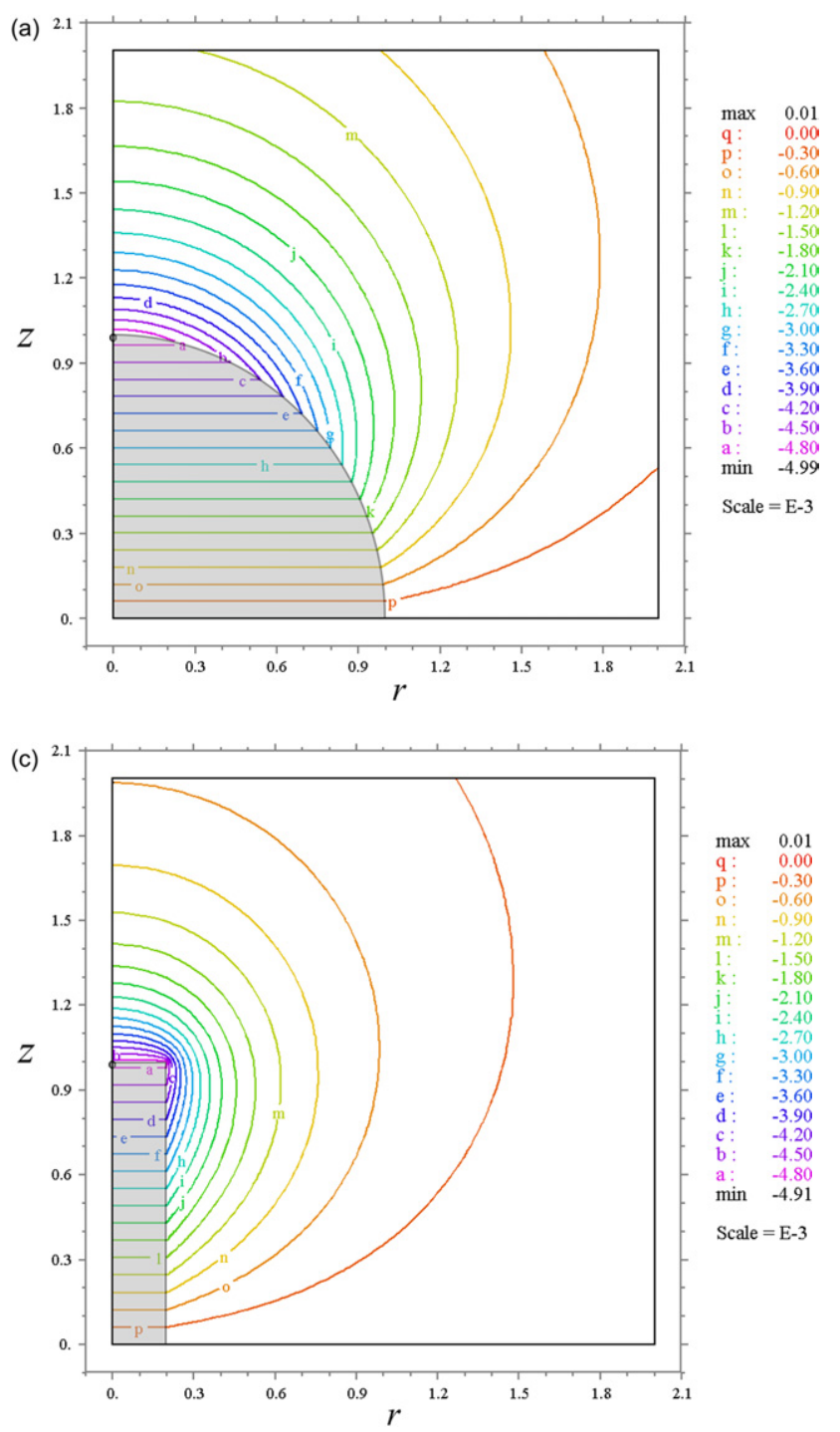

Figure 2. Numerically simulated electrical potential induced in the particle with the background applied field subtracted for (a) sphere, $(b)$ ellipsoid, $(c)$ cylinder and $(d)$ erythrocyte. The simulations were pseudo-three-dimensional in cylindrical coordinates $r$ and $z$ neglecting the angular rotation. Only the upper half of the simulation space is shown.

particles with the symmetry axis aligned with a uniform field $\boldsymbol{E}_{\mathrm{o}}$. The geometry is then pseudo-three-dimensional and can be solved in cylindrical polar coordinates $(r, \varphi, z)$, ignoring the $\varphi$ component while solving the field solutions and then incorporating it as a coefficient at the integration stage.

Figure 1(c) shows a schematic representation of the first three linear multipoles: the dipole, the quadrupole and the octupole. The electrical potential of the linear multipoles is given by the general equation [21-23]:

$$
\phi=\frac{p_{n}}{4 \pi \varepsilon r^{n+1}} P_{n}(\cos \theta),
$$

where $\varepsilon$ is the permittivity and $P_{n}(\cos \theta)$ are the Legendre polynomials, the first three of which are

$$
\begin{gathered}
P_{1}=\cos (\theta), \quad P_{2}=\frac{1}{2}\left(3[\cos (\theta)]^{2}-1\right), \\
P_{3}=\frac{1}{2}\left(5[\cos (\theta)]^{3}-3 \cos (\theta)\right), \ldots,
\end{gathered}
$$

where $\theta$ here represents the angle in spherical polar coordinates as indicated by figure $1(b)$. Because the multipoles are orthogonal, we can extract each multipolar term from the solution. For our system, comprising a single particle under the applied electric field, the electrical potential in the system is

$$
\phi_{\text {total }}=\phi_{\text {applied }}+\phi_{\text {particle }}
$$

where $\phi_{\text {imposed }}=-E_{\mathrm{o}} r \cos \theta$ is the potential due to the applied electric field and $\phi_{\text {particle }}$ is the potential due to the particle, given by

$$
\phi_{\text {particle }}=\sum_{n=1}^{\infty} \frac{p_{n}}{4 \pi \varepsilon r^{n+1}} P_{n}(\cos \theta) .
$$

The orthogonality of the Legendre polynomials [23] can be used to extract the multipoles from any arbitrary simulation or calculation of the electric field in the system. The linear multipoles are obtained from the equation

$$
p_{n}=4 \pi \varepsilon R_{\mathrm{t}}^{n+1} \frac{2 n+1}{2} \int_{0}^{\pi} \phi_{R_{\mathrm{t}}} P_{n}(\cos \theta) \sin \theta \mathrm{d} \theta,
$$




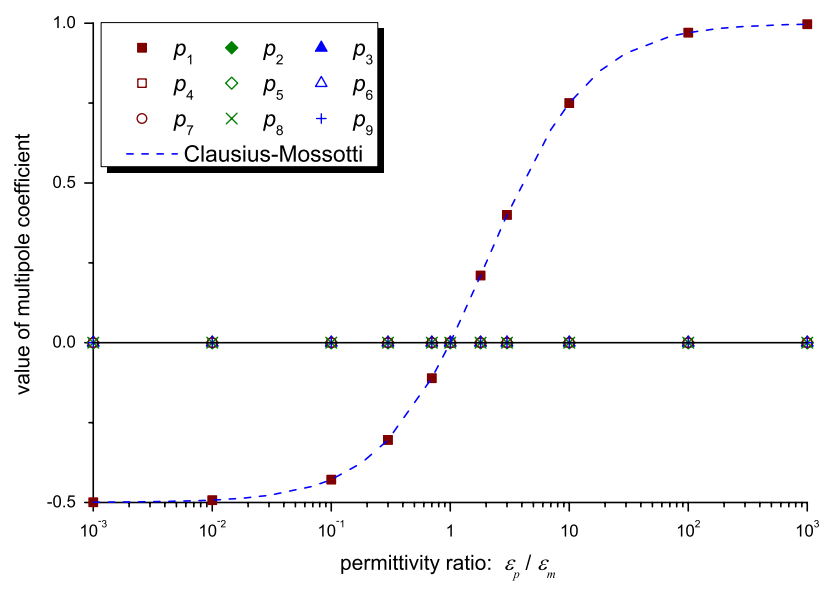

Figure 3. Results of the integration for the spherical particle as well as the analytical value given by the Clausius-Mossotti factor. The values for the first 9 multipole moments calculated numerically are plotted as symbols against the ratio of particle permittivity to fluid medium permittivity $\varepsilon_{\mathrm{p}} / \varepsilon_{\mathrm{m}}$. Only the dipole moment is non-zero and matches well with the analytical values which are plotted as a line.

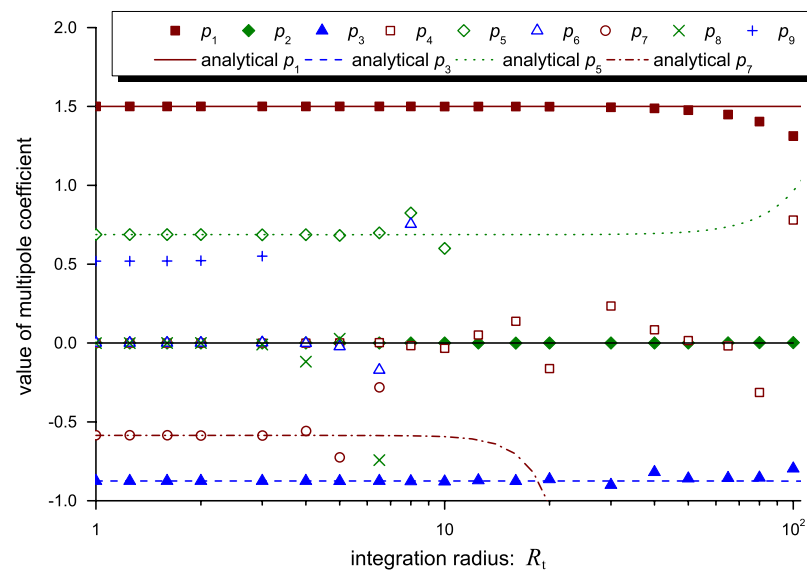

Figure 4. Results of the integration for the bisected sphere as well as the analytical calculations for the 1st, 3rd, 5th and 7th order moments. The values for the first 9 multipole moments calculated numerically are plotted as symbols and the analytical values as lines against the radius of integration.

where $\phi_{R_{\mathrm{t}}}$ is the electrical potential due to the particle on the spherical surface given by $r=R_{\mathrm{t}}$, which is determined from the numerical solution, $\phi_{\mathrm{FEM}}$, by subtracting the potential from the applied field:

$$
\phi_{R_{\mathrm{t}}} \equiv\left[\phi_{\mathrm{FEM}}-\phi_{\text {applied }}\right]_{r=R_{\mathrm{t}}} .
$$

For the finite element analysis, we use a commercial software package: FlexPDETM (PDE Solutions, Sunol, CA, USA).

\section{Results and discussion}

Simulations were performed for a range of particle shapes, as indicated schematically in figure $1(a)$. The problem is a simple solution of Poisson's equation for the electrical potential, $\phi$, with the free charge in the system set to zero:

$$
\nabla \cdot\left(\varepsilon_{\mathrm{r}} \varepsilon_{\mathrm{o}} \nabla \phi\right)=0,
$$

where $\varepsilon_{\mathrm{o}}$ is the permittivity of free space and $\varepsilon_{\mathrm{r}}$ is the relative permittivity of the different materials in the system. For each particle type, the electrical potential was numerically simulated for a range of particle permittivities relative to a fixed fluid permittivity of $1000 \varepsilon_{0}$, to model the effect of changing polarisabilities. The effective multipole moments were then calculated by integration of equation (4) for a range of different integration radii $R_{\mathrm{t}}$.

\subsection{Description of the test particles and electrical potential simulations}

The first test case, a simple homogeneous spherical particle with radius $a=1$, which has an analytical solution for the electrical potential and the dipole moment, can be used for direct comparison with our results. The electrical potential due to the particle only, i.e. $\phi_{\mathrm{FEM}}-\phi_{\text {applied }}$, for the case of a more polarisable particle $\left(\varepsilon_{\text {r,particle }} \gg \varepsilon_{\text {r,fluid }}\right)$ is shown in figure 2(a) for one-half of the particle.

The second test case is the bisected spherical void with the upper surface having a potential $\phi=1$ and lower surface $\phi=-1$ and, in this case, no applied electric field. The electrical potential outside the spherical void has a complete spectrum of odd numbered moments: $n=1,3,5, \ldots$ This test case allows us to establish the range of values of integration radius $R_{\mathrm{t}}$ for the determination of each multipole moment.

The third test case is ellipsoidal particles, characterised by three semimajor axes, $a_{1}, a_{2}$ and $a_{3}$ [21]. Axi-symmetric ellipsoids have the restriction that $a_{2}=a_{3}$, and $a_{1}$ is aligned along the direction of the electric field, the $z$-axis in this paper. The fractional relationship $e=a_{2} / a_{3}$ is referred to as the eccentricity of the ellipsoid, with a sphere being the particular case of $e=1$. The cases $e>1$ and $e<1$ are, respectively, prolate and oblate spheroids. In this paper, we simulated ellipsoidal particles with eccentricities of $10: 1,5: 1,2: 1$, $1: 2,1: 5$ and $1: 10$, keeping the largest radius equal to 1 in all cases. Figure $2(b)$ shows the solution for a more polarisable ellipsoidal particle with eccentricity $5: 1$.

The fourth test case is cylinders of height equal to $a_{1}$ and radius equal to $a_{2}$, so that the eccentricity factor $e=a_{2} / a_{3}$ can be used to compare cylinders with ellipsoids. In the literature, cylindrical particles are often modelled as ellipsoids. [28]. We have simulated cylinders with eccentricities of $10: 1,5: 1$, $2: 1,1: 2,1: 5$ and $1: 10$, keeping the largest radius equal to 1 in all cases. Figure 2(c) shows the solution for a more polarisable cylindrical particle with eccentricity $5: 1$.

The final test case is a model for the erythrocyte, the RBC, shown schematically in figure 1(a) with a cross-section. The shape is that of a rounded disc with a dimple on either side. The shape used in the simulation is an approximation to the relative sizes of a RBC, normalised to having a radius of 1 . An oblate ellipsoid with the same eccentricity was simulated for comparison.

\subsection{Spherical particle}

The dipole moment of a spherical particle is given by $[5,6,12,13]$

$$
\boldsymbol{p}_{1}=4 \pi a^{3} \varepsilon_{\mathrm{m}} \varepsilon_{\mathrm{o}} \operatorname{Re}\left[f_{\mathrm{CM}}\right] \boldsymbol{E}_{\mathrm{o}},
$$



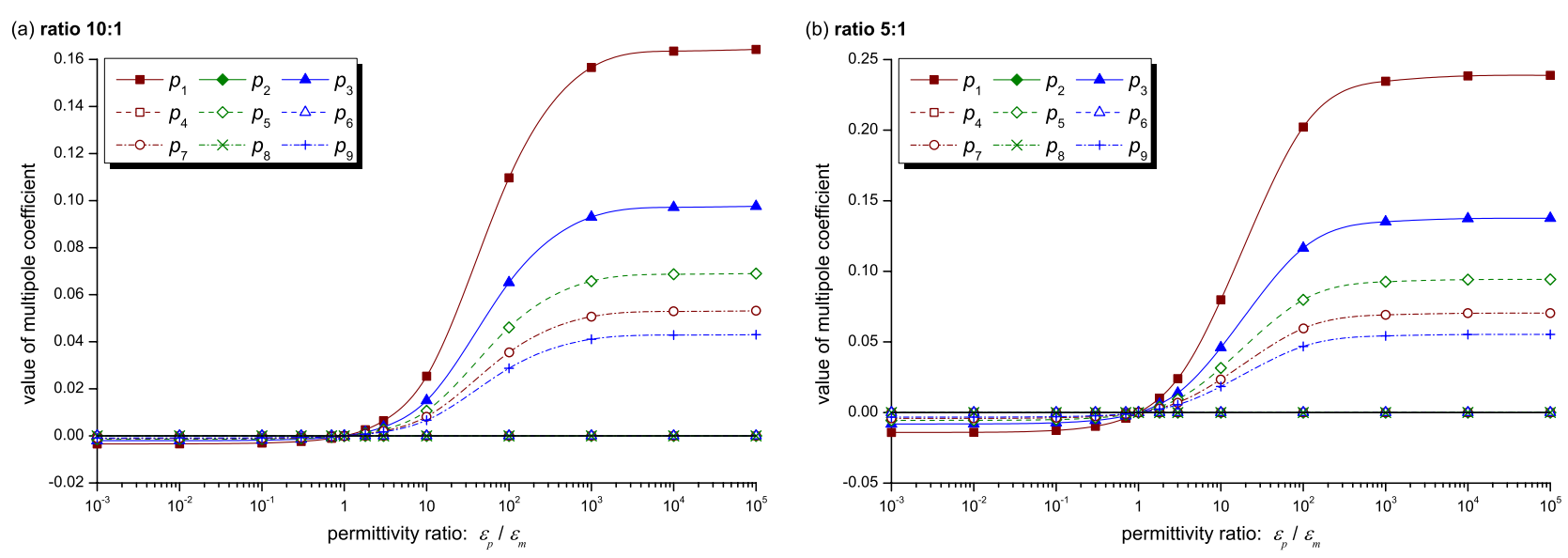

(c) ratio $2: 1$
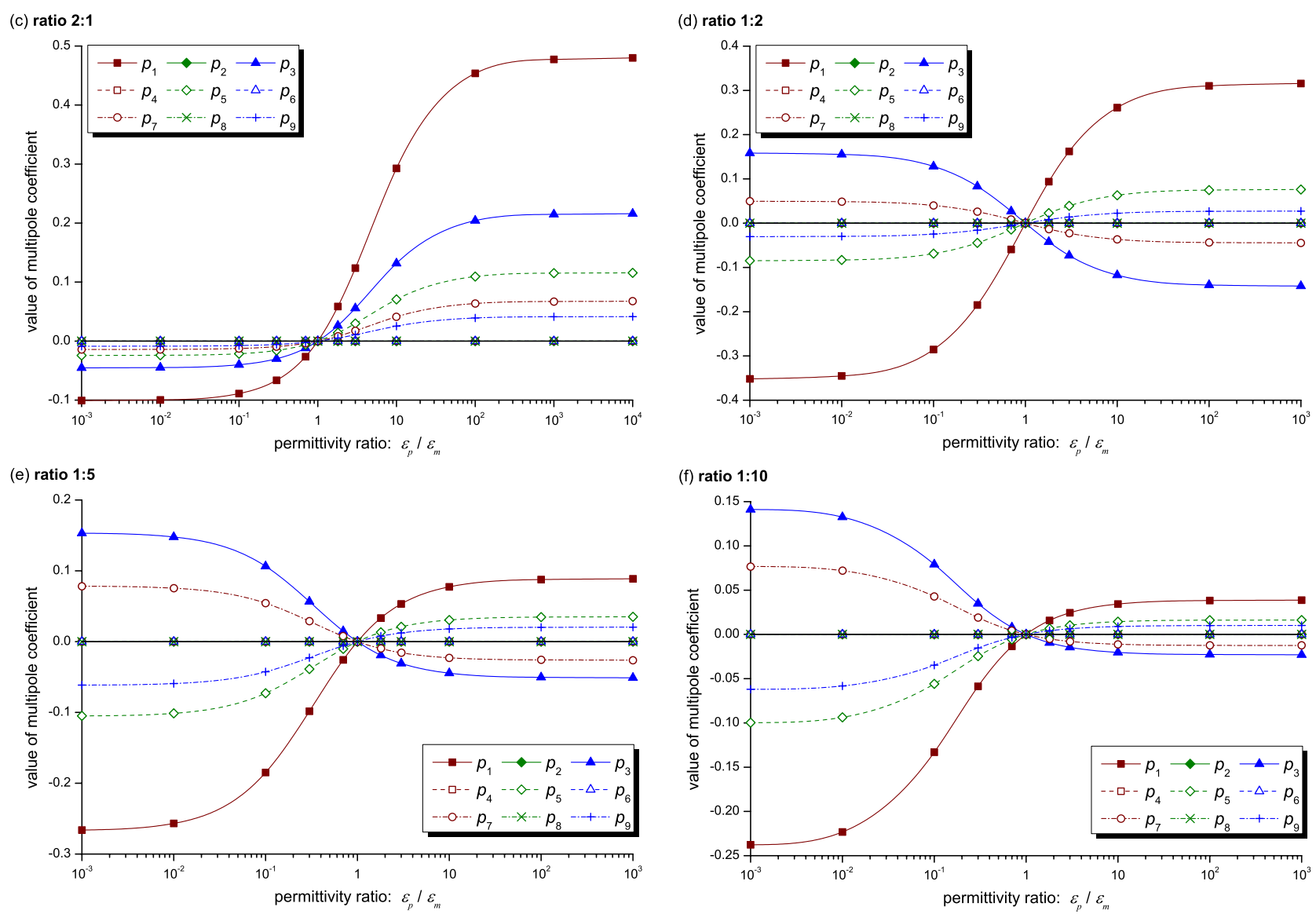

Figure 5. Results of the integration for ellipsoidal particles of different aspect ratios (eccentricity) $a_{1} / a_{2}$. The values for the first 9 multipole moments calculated numerically are plotted as symbols against the ratio of particle permittivity to fluid medium permittivity $\varepsilon_{\mathrm{p}} / \varepsilon_{\mathrm{m}}$.

where $a$ is the radius of the particle, $\varepsilon_{\mathrm{m}}$ is the relative permittivity of the suspending fluid, $\operatorname{Re}[\ldots]$ indicates the real part of and $f_{\mathrm{CM}}$ is the Clausius-Mossotti factor given by

$$
f_{\mathrm{CM}}=\frac{\tilde{\varepsilon}_{\mathrm{p}}-\tilde{\varepsilon}_{\mathrm{m}}}{\tilde{\varepsilon}_{\mathrm{p}}+2 \tilde{\varepsilon}_{\mathrm{m}}},
$$

where the tilda indicates complex permittivity: $\tilde{\varepsilon}=\varepsilon_{\mathrm{r}} \varepsilon_{\mathrm{o}}-\mathrm{i} \sigma / \omega$ where $\sigma$ is the conductivity, $\omega=2 \pi f$ is the angular frequency and $f$ is the frequency. The Clausius-Mossotti factor describes the frequency variation of the dipole moment and is bounded by -0.5 and +1 . For the purpose of testing the method, we are going to ignore the complex part of the permittivity.
Figure 3 shows the values of the first nine multipoles calculated from the integration of equation (4) around a spherical surface with radius $R_{\mathrm{t}}=2$. The results are in good agreement with the analytical solution for the dipole moment with the Clausius-Mossotti factor given by the dashed line. To within computational accuracy, the higher order moments are zero.

\subsection{Bisected sphere}

The purpose of the bisected sphere simulation is to determine the effective range of $R_{\mathrm{t}}$ over which the integration can be performed accurately. With the radius of the sphere set to 1 , 
Numerical determination of the effective moments of non-spherical particles

the first three moments can be calculated to an accuracy of $9.6 \%$ out to a radius of 100 . The symbols in figure 4 indicate the values extracted from the FEM simulations while the solid lines indicate the analytically calculated values. Owing to the significantly more rapid decrease in the higher order moments with radius and the fixed numerical error, the range of $R_{\mathrm{t}}$ over which these higher order terms can be determined is much smaller. In fact, the moments can be calculated at a larger value of $R_{\mathrm{t}}$ analytically than numerically. The even moments are all zero as expected from the odd symmetry of the problem. The calculations indicate that the moments up to order 9 can be calculated out to a value of $R_{\mathrm{t}}=3$. For comparison, the numerical error in the simulation indicated by the software had a maximum value of $0.007 \%$. The numerical error determined by comparing the solution of the field around the sphere with the analytically calculated field was around $0.01 \%$. In conclusion, if only the dipole and octupole are required, the integration of equation (4) can be performed up to $R_{\mathrm{t}}=100$. If higher order moments are required, then the value of $R_{\mathrm{t}}$ must be as small as possible while still enclosing the particle.

\subsection{Ellipsoids}

Figure 5 shows the calculated moments up to order 9 for six ellipsoids with different eccentricities. There are several trends that can be identified in the results. For $e>1$, all moments have the same sign, with the higher order moments becoming more significant as the eccentricity increases. A similar trend is indicated for $e<1$, but with each successive odd moment alternating in sign. If the eccentricity is bigger than 1 , the values for the moments are larger when the particle permittivity is greater than the fluid permittivity for the opposite case. If the eccentricity is much less than 1, the larger values are found when the particle permittivity is less than the fluid.

An analytical representation for the dipole moment of an ellipsoid can be found in the literature [21, 28], giving an equivalent factor which includes elements of the particle volume:

$$
p_{\mathrm{ELL}}=\frac{a_{1} a_{2} a_{3}}{3} \frac{\varepsilon_{\mathrm{p}}-\varepsilon_{\mathrm{m}}}{\varepsilon_{\mathrm{m}}+A_{n}\left(\varepsilon_{\mathrm{p}}-\varepsilon_{\mathrm{m}}\right)},
$$

where the depolarising factor $A_{n}$ is

$$
A_{n}=\frac{a_{1} a_{2} a_{3}}{2} \int_{0}^{\infty} \frac{\mathrm{d} s}{\left(s+a_{n}^{2}\right) \sqrt{\left(s+a_{1}^{2}\right)\left(s+a_{2}^{2}\right)\left(s+a_{3}^{2}\right)}}
$$

and $s$ is a dummy integration variable. Figure 6, comparing the numerically calculated dipole moment with the analytical expression for the six different ellipsoids, demonstrates very good agreement.

\subsection{Cylinders}

Figure 7 shows the calculated moment values for a cylinder with equal height and diameter demonstrating that the higher order moments are non-zero and the dipole is different than from that of a sphere. Figure 8 shows the calculated moments for the six different cylindrical particles, showing similar behaviour to that of ellipsoids. The main difference is that

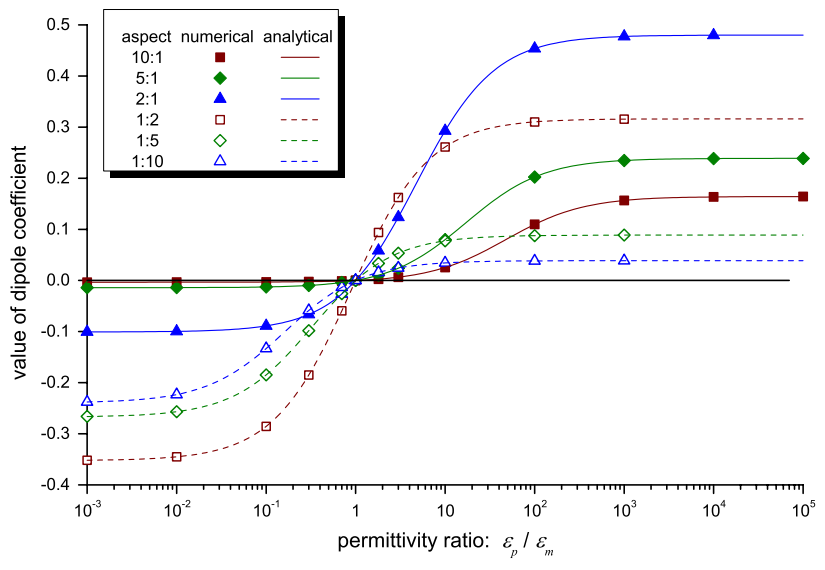

Figure 6. Calculated dipole moments for ellipsoidal particles for the six different particle eccentricities plotted as symbols against the ratio of particle permittivity to fluid medium permittivity $\varepsilon_{\mathrm{p}} / \varepsilon_{\mathrm{m}}$. Also plotted are the analytical values obtained from equation (9) as lines demonstrating perfect agreement.

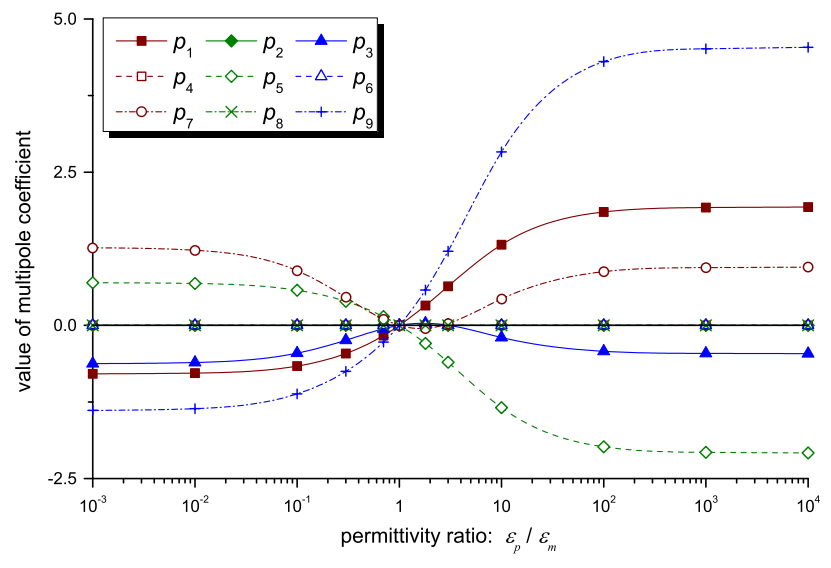

Figure 7. Results of the integration for cylindrical particles with an aspect ratio of $1: 1$, with the first 9 multipole moments plotted as symbols against the ratio of particle permittivity to fluid medium permittivity $\varepsilon_{\mathrm{p}} / \varepsilon_{\mathrm{m}}$.

the value of eccentricity for which the moments change from having the same sign to alternating is larger than $2: 1$ rather than being $1: 1$ as for ellipsoids. Again for values of the eccentricity much larger or much smaller than 1 , the higher order moments are comparable in size to the dipole.

In the literature, an ellipsoidal approximation is sometimes made for cylindrical particles. Figure 9 shows a comparison of the dipole moments for the six different eccentricities of cylinders shown by the symbols and the data for the ellipsoids with matching values of radii and eccentricity shown by the solid lines. As can be clearly seen, using an ellipsoid with the same axial radii as a given cylindrical particle is a poor approximation with the discrepancy up to $50 \%$ and a different variation with the ratio of permittivities.

\subsection{Erythrocyte}

Figure 10 shows the calculated odd moments for the erythrocyte shape indicated by the symbols and same moments for a matching sized oblate ellipsoid. The agreement is reasonable for each of the moments and could potentially be 

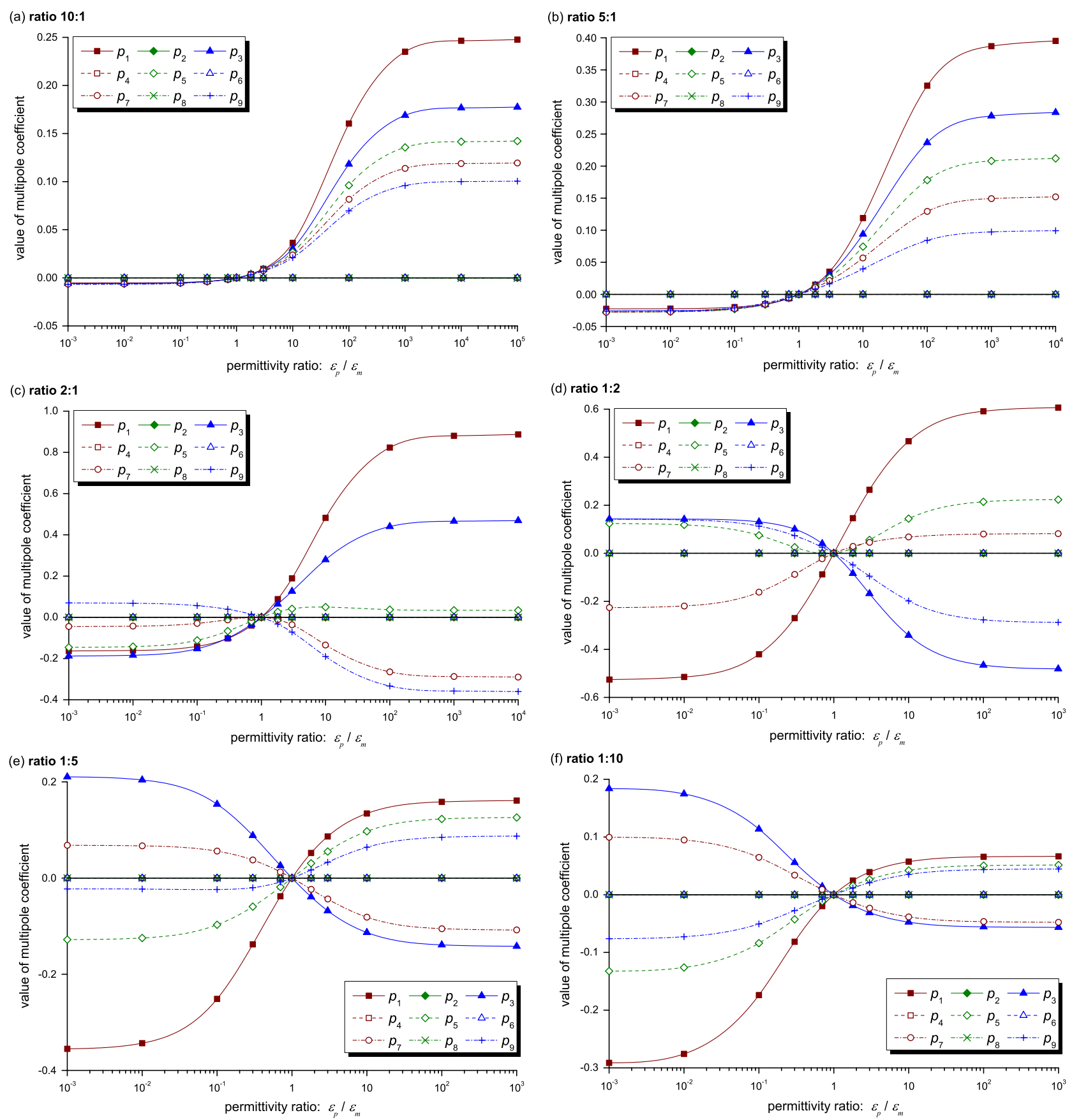

Figure 8. Results of the integration for cylindrical particles of different aspect ratios (eccentricity) $a_{1} / a_{2}$. The values for the first 9 multipole moments calculated numerically are plotted as symbols against the ratio of particle permittivity to fluid medium permittivity $\varepsilon_{\mathrm{p}} / \varepsilon_{\mathrm{m}}$.

improved by matching the volumes of the two particles rather than the dimensions.

\section{Conclusion}

A method has been presented for determining the dipole and other higher order moments for any cylindrically symmetric particle. This method has been tested on particle shapes having analytical representations, including prolate and oblate spheroids, truncated cylinders and an indented oblate spheroid. The moment calculations for prolate and oblate spheroids give results that are in very close agreement with theoretical values obtained directly from analytical solutions. Results for truncated cylinders, which are of possible interest in modelling proteins and self-assembled microstructures, are in poor agreement with similarly chosen spheroidal shapes, suggesting that using the latter to represent the former is not accurate. On the other hand, the indented oblate spheroid seems to provide fairly good agreement with a regular oblate spheroid.

The next task in this work is to extend the moment extraction technique to the general multipoles (spherical harmonics), which can influence forces and torques exerted on cells in planar quadrupolar traps and multilayer structures, when particle size becomes comparable to the dimensions 


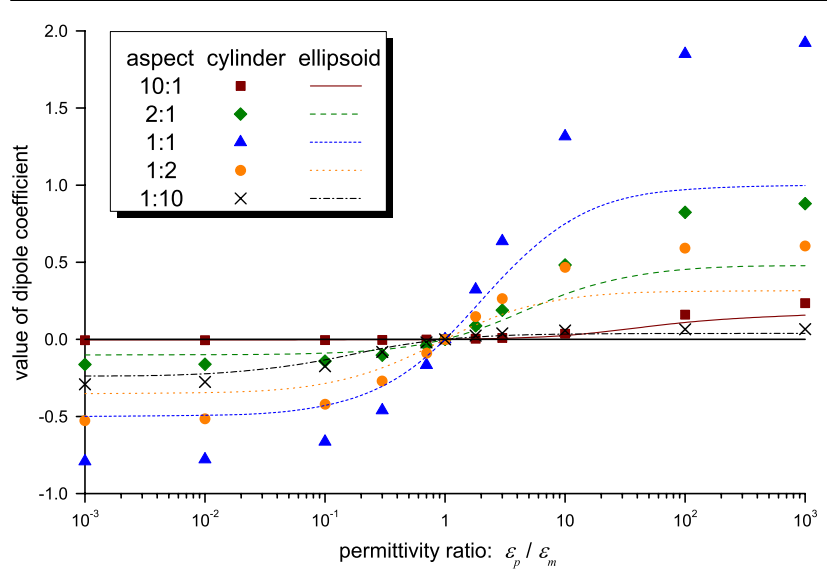

Figure 9. Comparison of the dipole moments for ellipsoidal particles (lines) and cylindrical particle (symbols). The values of the axial radii for the two different particle shapes matched in each case. The values for the different particles do not agree.

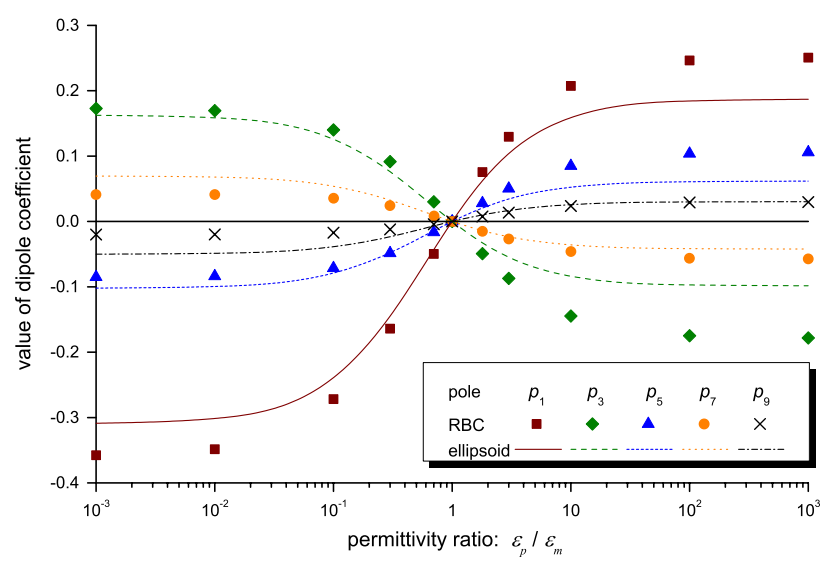

Figure 10. Comparison of the first 5 odd moments for the RBC (symbols) and a matching sized oblate ellipsoidal particle (lines), demonstrating reasonable agreement. The values are plotted as symbols against the ratio of particle permittivity to fluid medium permittivity $\varepsilon_{\mathrm{p}} / \varepsilon_{\mathrm{m}}$.

of the electrode structure. It has been established that the quadrupolar moment can have a significant influence on the net DEP force [29], and further development of our multipole extraction method may lead to simplified force and torque predictions in microelectrode structures.

\section{Acknowledgments}

NGG would like to thank the Royal Academy of Engineering, the Engineering and Physical Sciences Research
Council (EPSRC), UK, and the School of Electronics and Computer Science for funds to support this work. TBJ would like to thank the National Science Foundation (USA) as well as the EPSRC for a EPSRC-funded Visiting Fellowship at University of Southampton (2005).

\section{References}

[1] Pethig R and Kell D B 1987 Phys. Med. Biol. 32933

[2] Foster K R and Schwan H P 1989 Crit. Rev. Biomed. Eng. 17 25

[3] Schwan H P 1999 Ann. New York Acad. Sci. 8731

[4] Feldman Y, Ermolina I and Hayashi Y 2003 IEEE Trans. Dielectr. Electr. Insul. 10728

[5] Jones T B 1995 Electromechanics of Particles (Cambridge, UK: Cambridge University Press)

[6] Morgan H and Green N G 2003 AC Electrokinetics: Colloids and Nanoparticles (Baldock, Herts, UK: Research Studies Press)

[7] Fricke H 1931 Physics 1106

[8] Cole K S 1932 J. Gen. Physiol. 15641

[9] Asami K 2002 Prog. Polym. Sci. 271617

[10] Gawad S, Cheung K, Seger U, Bertsch A and Renaud P 2004 Lab Chip 4241

[11] Cheung K, Gawad S and Renaud Ph 2005 Cytometry A 65124

[12] Pohl H A 1978 Dielectrophoresis (Cambridge, UK: Cambridge University Press)

[13] Pethig R 1996 Dielectrophoresis: using inhomogeneous AC electrical fields to separate and manipulate cells Crit. Rev. Biotechnol. 16 331-48

[14] Gimsa J, Marszalek P, Loewe U and Tsong T Y 1991 Biophys. J. 60749

[15] Wang X-B, Huang Y, Becker F F and Gascoyne P R C 1994 J. Phys. D: Appl. Phys. 271571

[16] Fuhr G, Schnelle T, Muller T, Glasser H, Lisec T and Wagner B 1995 Sensors Mater. 7131

[17] Goater A D and Pethig R 1998 Parasitology 116177

[18] Arnold W D and Zimmermann U 1982 Naturwissenschaften 69297

[19] Ziervogel H, Glaser R, Schadow D and Heymann S 1986 Biosci. Rep. 6973

[20] Hölzel R. 1997 Biophys. J. 731103

[21] Stratton J A 1941 Electromagnetic Theory (New York: McGraw-Hill)

[22] Lorrain P, Corson D R and Lorrain F 1987 Electromagnetic Fields and Waves 3rd edn (New York: WH Freeman and $\mathrm{Co}$ )

[23] Jackson J D 1999 Classical Electrodynamics 3rd edn (New York: Wiley)

[24] Washizu M and Jones T B 1994 J. Electrostatics 33187

[25] Jones T B and Washizu M 1996 J. Electrostatics 37121

[26] Washizu M and Jones T B 1996 J. Electrostatics 38199

[27] Munoz San Martin S, Sebastian J L, Sancho M and Miranda J M 2003 Phys. Med. Biol. 481649

[28] Morgan H and Green N G 1997 J Electrostatics 42279

[29] Schnelle T, Müller T and Fuhr G 1999 Biomethods 10417 